\title{
Video Processing for Remote Respiration Monitoring
}

\author{
Davide Alinovi \\ Department of Engineering and Architecture, University of Parma, Italy \\ Advisor: Riccardo Raheli \\ Date and location of PhD defense: 14th March 2017, University of Parma, Italy
}

Received 14th July 2017; accepted 8th October 2017

\begin{abstract}
Monitoring of vital signs is a key tool in medical diagnostics. Among fundamental vital parameters, the Respiratory Rate (RR) plays an important role as indicator of possible pathological events. For this reason, respiration needs to be carefully monitored in order to detect potential signs indicating possible changes of health conditions. In this work, novel techniques for the visualization and analysis of respiration by remote and non-invasive video monitoring, based on the study of breathing-related movements, are proposed. The lack of large video databases, associated with clinical data, essential for performance evaluation and optimization of the video processing-based algorithms, is also addressed; statistical models of respiration and apnea events are proposed together with proper simulators, useful to test the remote monitoring algorithms.
\end{abstract}

\section{Introduction}

Efficient monitoring of vital signs, intended as the measurement by appropriate devices of signals related with human bodily functions, has the fundamental task of helping clinicians to assess the health conditions of a patient and check for the possible outbreak and evolution of physical disorders. Main vital signs monitored by healthcare providers include, e.g., heart rate, blood pressure, breathing rate and body temperature.

One of the main physiological tasks that needs to be carefully monitored in patients is the respiration. Among parameters used to control the efficiency of the respiration system, the Respiratory Rate (RR) plays a key role. In fact, anomalous rates can be significant indicators of a hidden pathological condition. Specifically, they could be useful to predict life-threatening events or reveal hazardous absence of respiration, such as an apnea event. These events are defined as the cessation of respiratory airflow and can be also caused by life-threatening diseases, that need timely treatments; moreover, in newborns, there may be a risk of Sudden Infant Death Syndrome (SIDS). For this reason, the RR may be continuously monitored.

Nowadays, the gold-standard methods for respiration monitoring mainly employed in clinical environments are based on devices that require a direct connection to the patient's body. These devices cannot always be used in home environments or applied for telemedicine or in eHealth contexts. Furthermore, the direct connection of the sensors to the patient, makes these systems quite uncomfortable, particularly if applied to infants.

Correspondence to: davide.alinovi@unipr.it

Recommended for acceptance by Anjan Dutta and Carles Sánchez

DOI: https://doi.org/10.5565/rev/elcvia.1124

ELCVIA ISSN: 1577-5097

Published by Computer Vision Center / Universitat Autònoma de Barcelona, Barcelona, Spain 
In recent times, the research and development of portable, comfortable and even contactless equipments for the monitoring of respiration and other vital signs experienced a dramatic growth, also thanks to the miniaturization and drop of price of the sensors involved. One of the most appealing methods employed for monitoring tasks and to create possible automatic diagnostic tools is based on image and video processing.

The main focus of this work is the development and analysis of novel video processing-based algorithms for the monitoring of respiration, also addressing the lack of large video databases, necessary for their design, implementation, testing and optimization [1].

\section{Remote Monitoring of Respiration}

Motion-related variations in video signals may be recognized by video processing-based algorithms to identify movements related to respiration. Information extracted by such algorithms can be employed for the detection of specific breathing disorders, in particular apneas, and the estimation of the RR.

Methods Motion related to respiration is difficult to detect as temporal variation in video signals, especially in newborns. In fact, very small changes have to be detected in order to identify the breathing effort and estimate the RR. For this purpose, three algorithms are proposed, tested and calibrated for the monitoring of newborns, who need to be carefully overseen. The first solution exploits a motion magnification algorithm, also known as Eulerian Video Magnification (EVM), with the purpose to enhance and highlight small periodic movements so that they become detectable. Applying the EVM algorithm as a pre-processing technique, the extraction of an average motion signal describing respiration-related movements, followed by periodicity analysis based on Maximum Likelihood (ML) methods, leads to the detection of possible lack of breathing movements [2, 3]. The motion magnification technique is then improved by embedding it in the algorithm for the extraction of motion signal and reinforce the estimation of periodicity. The newly proposed algorithm is tested on regularly breathing newborns to measure the RR estimation performance [4].

The previously described techniques make both use of the ML approach for the estimation of the fundamental frequency related with respiration applied to a suitable monodimensional signal. Exploiting the properties and the advantage of this approach and relying on the fact that breathing involves quasi-periodic movements, a new proposed algorithm employs a generalized model of pixel-wise periodicity and applies the ML criterion directly on raw video streams [5, 6]. The system first selects suitable Regions of Interest (ROI) mainly affected by respiratory movements: the obtained ROI are then jointly analyzed for the estimation of the RR of the framed patient. Furthermore, a large motion detection algorithm is applied in order to exclude the RR affected by movements unrelated to respiration.

Results Employing the solution proposed in $[2,3]$ to detect apnea events, the performance is assessed in terms of sensitivity and specificity, using the polysomnography as ground truth. The performance of the embedded motion magnification algorithm [4] in RR estimation is then considered, showing a significant improvement with respect to the previous method. In Figure 1(a), an illustrative frame of the considered video sample is shown. In (b), a comparison of the pneumogram with the motion signal extracted by the video processing-based algorithm is presented-it shows a good agreement with the signal given by the medical device, considering that each respiratory act is composed of two main movements: inhalation and exhalation.

Finally, the last developed algorithm [5] can improve RR estimation performance with respect to the previous ones, thanks to a better management of the large motion of the patient and environmental changes.

\section{Models and Simulators of Respiration}

A significant problem which hinders the design and optimization of video processing-based monitoring systems is the lack of large video databases, associated with clinical data, obtained from real patients with respira- 


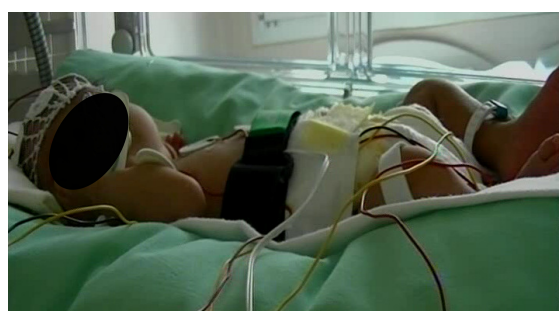

Camera framing a newborn patient.

(a)

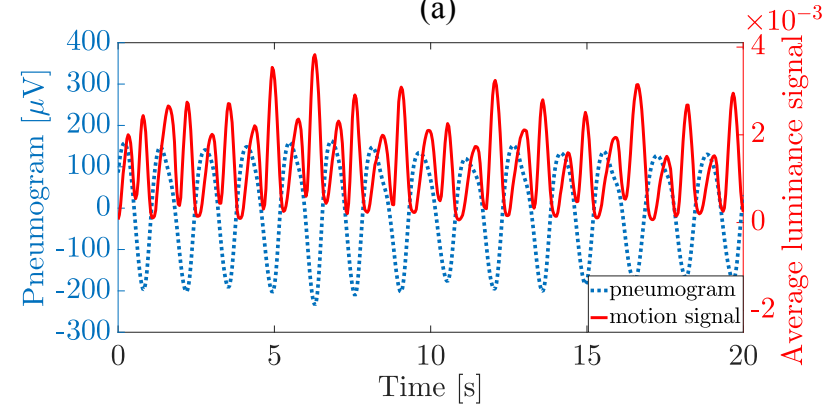

(b)

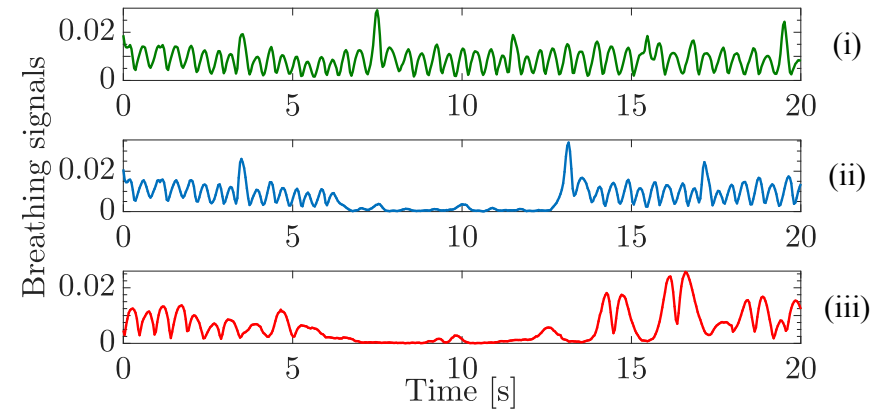

(i) regular breathing; (ii) simulated apnea; (iii) real apnea.

(c)

Figure 1: (a) Sample frame of a monitored newborn. (b) Example of breathing signal extracted by the video processing algorithm in [4] compared with the pneumogram. (c) Comparison on breathing signals of (ii) a simulated apnea, obtained from a regular breathing patient (i), with (iii) a real apnea.

tory disorders.

Methods A statistical model, based on Continuous-Time Markov Chains (CTMC), of the RR patterns of a patient is developed. First, a simple two-state model which statistically describes the occurrence of apneas in patients suffering from breathing disorder is introduced [7]. The obtained model is able to reproduce realistic patterns of the succession of regular breathing intervals and apnea events with a properly selected duration. This model is then extended to a multiple-state CTMC to represent complete and realistic breathing patterns, considering the variation of the RR of a patient over time [8].

Following this approach, suitable simulators based on these statistical models have been developed to test video-processing based algorithms. In particular, two simulators are introduced: software- and hardwarebased. The software simulator directly manipulates video recordings in order to introduce apnea events or create custom respiration patterns in a regularly breathing patient $[7,8]$. The hardware simulator consists of a manikin of a newborn, with a "moving chest" able to reproduce respiratory movements following the statistical models of breathing patterns [8].

Results The CTMC-based models are driven by real data extracted from monitored patients by a pneumographic device. The developed models are validated by comparing the statistical characteristics of the real cases with the synthetic ones, generated by the CTMCs models.

In Figure 1(c) a direct comparison between motion signals extracted from (ii) a simulated apnea and (iii) a real apnea from a child suffering from severe apnea events is depicted, showing the effectiveness of the apnea simulator. Moreover, in part (i) of Figure 1(c) the motion signal of the original video framing a regular breathing newborn from which the simulated apnea was obtained is also reported.

Finally, the two simulators are used to assess the performance of the previously proposed video processingbased algorithms, showing the usefulness of the simulators and the effectiveness of the algorithms in apnea detection and accuracy in RR estimation [8]. 


\section{Conclusion}

This work focuses on the development of algorithms for remote monitoring and analysis of breathing movement. Information extracted by video processing-based algorithms is then employed: first, for the detection of apneas and then for the estimation of the RR.

The problem concerning the lack of large video databases, necessary to design, implement and test video processing-based algorithms for respiration monitoring, is also discussed. A statistical model, based on CTMC, of the RR patterns of a patient is then proposed. Following this strategy, suitable simulators to test videoprocessing based algorithms are developed. Performance evaluation of the proposed video processing-based algorithms is so obtained, showing the effectiveness of both algorithms and the relevant simulators.

\section{References}

[1] D. Alinovi, "Video Processing for Remote Respiration Monitoring," Ph.D. Dissertation, Dept. Eng. and Archit., Univ. of Parma, Parma, Italy, Mar. 2017. [Online]. Available: http://hdl.handle.net/1889/3416.

[2] L. Cattani, D. Alinovi, G. Ferrari, R. Raheli, E. Pavlidis, C. Spagnoli, and F. Pisani, "A wire-free, noninvasive, low-cost video processing-based approach to neonatal apnoea detection," in Proc. 2014 IEEE Workshop Biometric Meas. and Syst. Security and Med. Appl. (BIOMS), Rome, Italy, Oct. 2014, pp. 6773. doi:10.1109/BIOMS.2014.6951538.

[3] L. Cattani, D. Alinovi, G. Ferrari, R. Raheli, E. Pavlidis, C. Spagnoli, and F. Pisani, "Monitoring infants by automatic video processing: a unified approach to motion analysis," Comput. Biol. Med. (Elsevier), vol. 80, pp. 158-165, Jan. 2017. doi:10.1016/j.compbiomed.2016.11.010. Link to supplementary multimedia material.

[4] D. Alinovi, L. Cattani, G. Ferrari, F. Pisani, and R. Raheli, "Spatio-temporal video processing for respiratory rate estimation," in Proc. 2015 IEEE Int. Symp. Med. Meas. and Appl. (MeMeA), Turin, Italy, May 2015, pp. 12-17. doi:10.1109/MeMeA.2015.7145164.

[5] D. Alinovi, G. Ferrari, F. Pisani, and R. Raheli, "Respiratory rate monitoring by maximum likelihood video processing," in Proc. 2016 IEEE Int. Symp. Signal Process. and Inf. Technol. (ISSPIT), Limassol, Cyprus, Dec. 2016, pp. 172-177. doi:10.1109/ISSPIT.2016.7886029.

[6] D. Alinovi and R. Raheli, "Extraction of periodic features from video signals," in Proc. Ninth IARIA Int. Conf. Advances Multimedia (MMEDIA), Venice, Italy, Apr. 2017, pp. 95-100. ISBN: 978-1-61208-548-7.

[7] D. Alinovi, L. Cattani, G. Ferrari, F. Pisani, and R. Raheli, "Video simulation of apnoea episodes," in Proc. 2015 IEEE Int. Conf. Multimedia and Expo Workshops (ICMEW), Turin, Italy, June 2015, pp. 1-6. doi:10.1109/ICMEW.2015.7169869.

[8] D. Alinovi, G. Ferrari, F. Pisani, and R. Raheli, "Markov chain modeling and simulation of breathing patterns," Biomed. Signal Process. Control (Elsevier), vol. 33, pp. 245-254, Mar. 2017. doi:10.1016/j.bspc.2016.12.002. Link to supplementary multimedia material. 